\title{
La vidange de la retenue de Pareloup en 1993: une étape-clef dans la vie du lac
}

\author{
Draining of Pareloup reservoir in 1993: a key phase in \\ the life of the lake
}

\section{G. Merle( $\left(^{*}\right)$, D. Mosnier(**), J.N. Tourenq(***)}

(*) Service Environnement, EDF Production Transport. 6, rue Ampère. B.P. 114. F-93203 SaintDenis cedex 1, France.

("*) Services et Ingénierie Sud Ouest, EDF Production Transport. 44, avenue de Muret.

F-31052 Toulouse cedex. France.

(**) Laboratoire d'Hydrobiologie, URA 695 CNRS, Université Paul Sabatier. 118, route de Narbonne. F-31062 Toulouse cedex, France.

Résumé. - La retenue de Pareloup qui existe depuis 1952 n'avait pas fait l'objet d'une vidange complète depuis 1962. Elle a été entièrement vidée en 1993 pour procéder à une inspection réglementaire des structures du barrage et à de nécessaires travaux d'entretien.

Les nombreuses études hydrobiologiques déjà réalisées sur le lac de Pareloup les 10 dernières années ont été mises à profit pour adapter au mieux la procédure de vidange au cycle biologique du lac et pour en réduire les impacts sur l'environnement.

De nombreux enseignements peuvent être tirés de celte vidange grâce au suivi particulièrement important qui a été fait de toutes les phases de l'opération. Les recherches se poursuivent après le nouveau remplissage de la retenue, sur les cours d'eau à l'aval et sur l'écosystème lacustre pour étudier les effets de la discontinuité créée par la vidange.

Mots-clés. - Retenue artificielle, vidange, suivi, physico-chimie, hydrobiologie, poissons.

Abstract. - Pareloup reservoir, created in 1952, had not been completely drained since 1962. This was done in 1993 for the purposes of regulation inspection of dam structures and to perform necessary maintenance.

The numerous hydrobiological studies already carried out on Pareloup in the last decade were drawn on to adapt the draining procedure as closely as possible to the biological cycle of the lake and to reduce environmental impact.

Many lessons are to be learned from this draining, thanks to the close monitoring of 
every phase of the operation. Now that the reservoir has been refilled, research is continuing on the downstream rivers and on the lake ecosystem, with a view to analysing the effects of the disruption created by the draining.

Key-words. - Artificial reservoir, draining, follow-up, physicochemical characteristics, hydrobiology, fish.

\section{LE CONTEXTE}

L'obligation légale d'un examen visuel direct et le nécessaire entretien des parties immergées, après 30 ans sans vidange de cet ouvrage âgé de quarante ans, ont conduit à réaliser en 1993 une vidange complète de la retenue de Pareloup.

Dès 1990, EDF a engagé une concertation avec l'Administration ainsi qu'une communication importante afin de préparer cette opération qui nécessitait, pour réaliser les travaux, une phase d'assec longue ( 3 mois) durant la période estivale et qui, par conséquent, allait avoir des répercussions importantes sur la Région compte tenu de l'importance touristique de ce lac pour l'Aveyron.
Deux commissions ont été créées par le Préfet, vue l'ampleur du dossier: une commission d'experts et une commission d'élus riverains. Au cours des réunions de ces commissions, l'ensemble des problèmes soulevés par la vidange a pu être débattu et EDF, à qui incombait la totalité des opérations techniques, a pu établir des plans d'action et prévoir les études nécessaires. Les incidences de la vidange sur les autres usagers de l'eau, sur l'environnement aquatique et sur le public ont été gérées en s'assurant le concours d'interlocuteurs compétents dans chaque domaine. L'ensemble des dispositions à respecter a été consigné dans l'arrêté préfectoral du 23 mars 1993 autorisant la vidange (tableau I).

Tableau I. - Principales valeurs à respecter données dans l'arrêté préfectoral du 23 Mars 1993 autorisant la vidange.

Table I. - Main values to be respected as specified in the official decree dated 23 March 1993 authorizing the draining.

\begin{tabular}{|c|c|c|c|}
\hline Paramètre & $\begin{array}{l}\text { Valeur } \\
\text { moyenne }\end{array}$ & $\begin{array}{c}\text { Valeur } \\
\text { maximale }\end{array}$ & Observations \\
\hline — & & 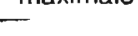 & $\therefore \ldots$. \\
\hline MES & $<3 g / 1$ & $8 \mathrm{~g} / \mathrm{l}^{*}$ & $\begin{array}{l}\text { - à ramener à } 5 \mathrm{~g} / \text { dans les } 2 \text { heures suivantes: } \\
\text { dilution par Bage si valeur instantanée }>3 \mathrm{~g} / \mathrm{l}\end{array}$ \\
\hline $\begin{array}{l}\text { Oxygène dissous } \\
\text { Teneur en } \mathrm{NH}_{4} \\
\text { Oxydabilité au } \mathrm{KMnO}_{4}\end{array}$ & $\begin{array}{l}>6 \mathrm{mg} / 1 \\
<1,0 \mathrm{mg} / 1\end{array}$ & $\begin{array}{l}>2 \mathrm{mg} / \mathrm{l} \\
<2,0 \mathrm{mg} / \mathrm{l} \\
10 \mathrm{mg} / \mathrm{l}^{* *}\end{array}$ & " en aval du confluent Vioulou/Viaur \\
\hline
\end{tabular}


De nombreux spécialistes dans des domaines techniques ou scientifiques ont participé à cette opération et une grande richesse d'informations a été recueillie. Cette note n'a pour ambition que de donner une image synthétique et non exhaustive des principaux points marquants de la vidange, les études particulières qui ont été menées à cette occasion faisant, elles, l'objet de communications scientifiques spécifiques dont plusieurs dans le présent numéro d'Hydroécologie Appliquée.

\section{LE DÉROULEMENT DE LA VIDANGE}

La retenue, à sa cote normale d'exploitation a une superficie de 1260 hectares et contient 169 millions de $\mathrm{m}^{3}$ d'eau (cf. présentation de l'amé- nagement du Pouget et du barrage de Pareloup, Energie Midi-Pyrénées, 1994)). Elle est placée sur le cours du Vioulou, affluent rive gauche du Viaur avec lequel it conflue à $10 \mathrm{~km}$ en aval du barrage de Pareloup.

L'évacuation des eaux ne peut se faire que progressivement pour limiter les nuisances vers l'aval et en utilisant les différentes possibilités d'évacuation disponibles (turbinage puis évacuation par le cours du Vioulou). Ceci demande en pratique plusieurs mois. On peut distinguer 4 phases principales durant la vidange (fig. 1).

\section{2-1 Une phase d'abaissement lent du plan d'eau (de Novembre 92 à juin 93)}

Durant cette phase:

- il n'y a pas de modification notoire de la qualité physico-chimique de

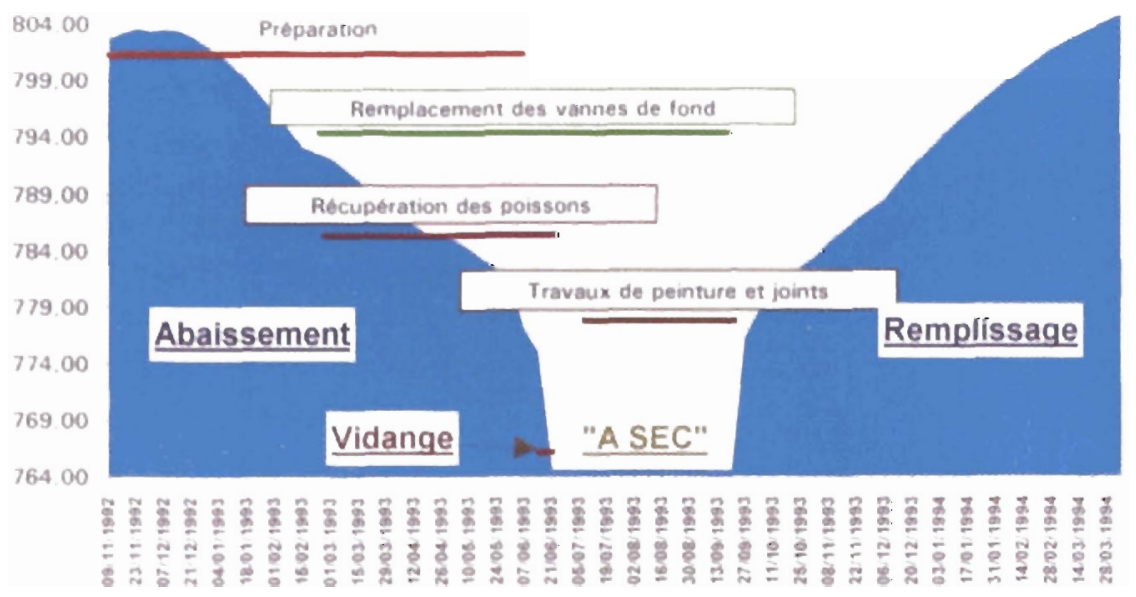

Fig. 1. - Phases principales de la vidange.

Fig. 1. - Main phases in draining of the reservoir. 
l'eau à l'exception d'un léger enrichissement en phosphore. Une vitesse très lente d'abaissement du plan d'eau par turbinage ne provoque pas d'entraînement important de sédiments et il s'agit d'une période où il n'y a pas de stratification thermique marquée d'où une assez grande homogénéité des paramètres de qualité d'eau notamment des teneurs en oxygène dissous,

- des analyses des sédiments permettent de connaître leur composition et de prévoir leur comportement au cours de la phase suivante de la vidange. Les sédiments de Pareloup sont pauvres en matière organique et donc peu consommateurs d'oxygène (Labroue et al., 1994)

- les poissons voient leurs zones de répartition diminuer et se concentrent progressivement. Des estimations du stock piscicole présent sont réalisées par pêches au filet et par écho-intégration (Richeux et al., 1994). Ceci donne début Juin 1993 un stock piscicole estimé à plus d'un million d'individus mais que l'on hésite à convertir en une valeur précise de biomasse compte-tenu du peu d'étalonnages existants. $\mathrm{La}$ fourchette estimée est de 60 à 100 tonnes qui, d'après les études menées par l'ENSAT et l'Université Paul Sabatier de Toulouse (UPS) depuis 1970 sur la répartition par espèces, se décomposent de la façon suivante pour 60 tonnes: 10 tonnes de carnassiers (brochets, sandres, perches), 30 tonnes de brèmes, 15 tonnes de gardons, les 5 tonnes restants étant ré- parties entre tanches, chevesnes, truites...

- la période est mise à profit pour faire des pêches scientifiques au filet (étude de l'état physiologique des poissons), des essais de pêche au lamparo, des concours de pêche,

- sur les zones découvertes, on réalise un épandage par hélicoptère de graines pour accélérer et renforcer la stabilité et l'imperméabilisation des sols en vue de limiter les entrainements lors d'orages. Ceci améliore aussi l'aspect visuel du lac et prépare les terrains pour les activités touristiques de remplacement,

- enfin on construit la pêcherie à l'aval du barrage. Le projet retenu consiste à la mise en place sur un ressaut naturel de la rivière d'une "prise d'eau par en dessous" à grilles très fines (espacement de $1 \mathrm{~cm}$ ) afin d'écrémer le poisson (fig. 2).

A la fin de cette première phase, il restait dans la retenue 1,3 millions de $\mathrm{m}^{3}$ d'eau renfermant la totalité des poissons. Ce volume est appelé "le culot ". Initialement prévue pour début juillet, la phase de vidange du culot qui se réalise en quelques jours par ouverture de la vanne de fond du barrage a été avancée (en accord avec le Préfet) au 14 juin pour tenir compte d'un échauffement assez rapide des eaux qui risquait d'être préjudiciable à la bonne conservation du cheptel piscicole. 


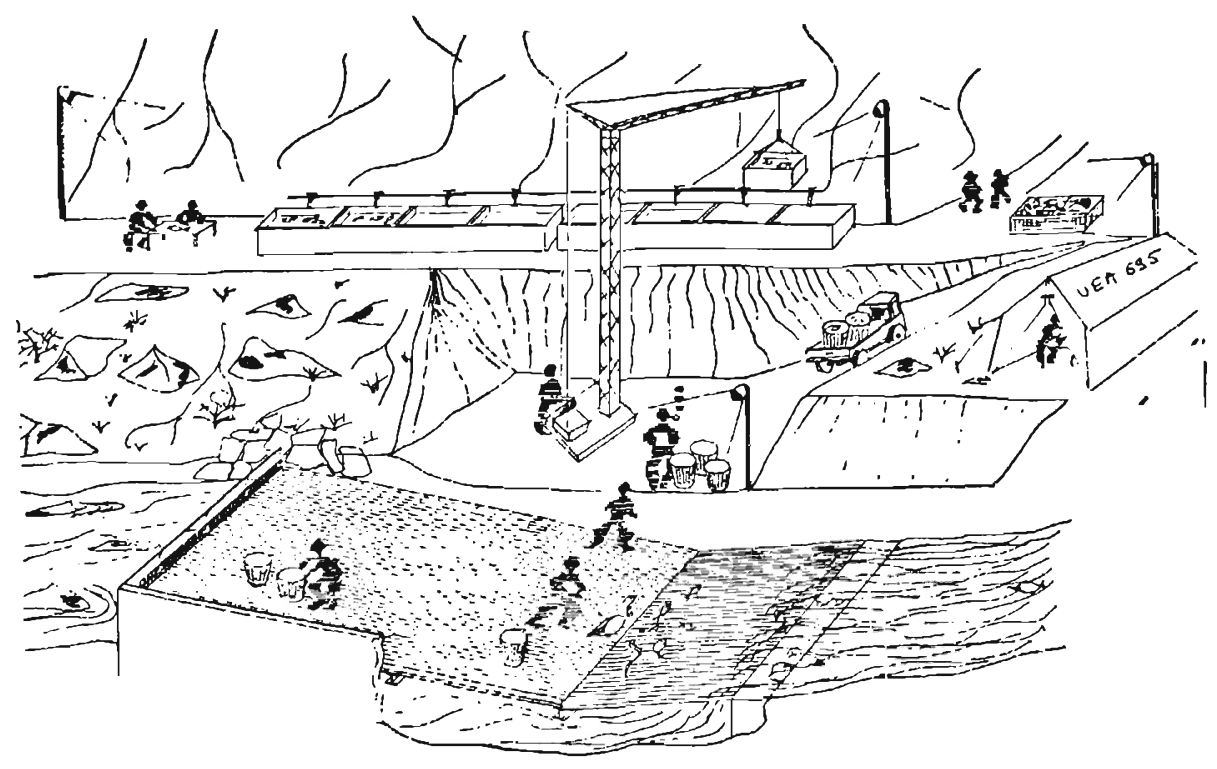

Fig. 2. - Shéma d'installation de la pêcherie.

Fig. 2. - Schema of the fishery installations.

\section{2-2. La vidange proprement dite}

Cette phase assez rapide s'est réalisée par ouverture de la vanne de fond et s'est poursuivie jusqu'à ce que le Vioulou retrouve son ancien lit au milieu de la retenue totalement asséchée.

\section{Elle a eu lieu du 14 au 17 juin} 1993.

La figure 3 donne l'allure de la courbe d'abaissement du plan d'eau à partir de l'ouverture des vannes de fond. On peut noter le rythme régulier de cet abaissement ( $11 \mathrm{~cm} /$ heure) hormis quelques oscillations au passage du culot final induites par des manœuvres de vannes rendues né- cessaires pour permettre le décolmatage des grilles de la pêcherie.

La vitesse d'abaissement du plan d'eau a été ajustée en permanence par le responsable de la vidange en modulant le débit des vannes de fond en fonction des informations obtenues grâce au suivi en temps réel de la qualité physico-chimique des eaux et aux observations concernant le comportement des poissons. Trois objectifs principaux devaient être en effet conciliés au mieux :

- évacuer l'eau du culot dans les conditions de respect des normes de qualité d'eau fixées par l'arrêté préfectoral,

- récupérer la totalité des poissons présents dans la retenue dont 


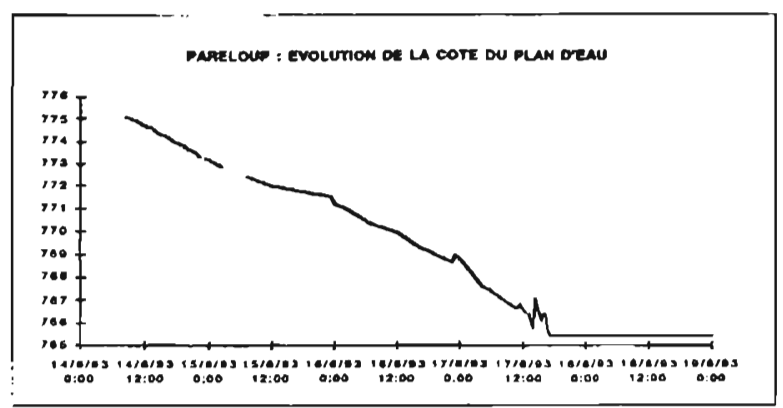

Fig. 3. - Courbe d'abaissement du plan d'eau à partir de l'ouverture des vannes de fond. Fig. 3. - Curve for drop in water level following opening of the bottom gates.

le plus possible vivants pour permettre les opérations de ré-alevinage,

- limiter en quantité et en durée le transfert des sédiments et composés polluants associés vers l'aval.

Pour atteindre ces objectifs, le responsable de la vidange était assisté d'experts hydrauliciens, biologistes, spécialistes des poissons. La procédure LIVRE (Limitation de l'Impact des Vidanges de REtenues, Cardinal, 1988) mise au point par EDF, Direction des Etudes et Recherches a été appliquée. Le suivi en temps réel des paramètres clés que sont la teneur en matières en suspension, en oxygène dissous, en fer, en manganèse a été réalisé par la Division Technique Génèrale (DTG) durant les 4 jours de la vidange en 6 points de mesure (Andrieu, 1993) répartis depuis la sortie de la vanne de fond jusqu'à $3 \mathrm{~km}$ en aval du confluent Vioulou-Viaur (fig. 4). Ce sont les résultats de ces mesures qui ont permis de guider les manceuvres d'évacuation des eaux et de décider du moment où il a été né- cessaire d'apporter un débit complémentaire d'eau claire par pompage dans la retenue de Bage pour diluer les eaux sortant des vannes de fond. Elles permettent aussi de faire un bilan de la qualité des eaux durant la vidange.

Pour compléter le dispositif de contrôle notamment vis-à-vis des stations d'eau potable situées plus à l'aval sur le Viaur, deux stations $A$ et $B$ de mesures physico-chimiques ont été placées à 4 et $38 \mathrm{~km}$ (Usine AEP de Thuriès), à l'aval de la confluence du Vioulou avec le Viaur. Les analyses et prélèvements ont été assurés par l'Agence de l'Eau Adour-Garonne et le Laboratoire d'Hydrobiologie (URA 695) de I'UPS (Simonet et al., 1993).

\section{Bilan des analyses effectuées}

\section{A) Matières en suspension totales}

Les prélèvements et analyses effectuées dans la retenue avant la vidange par le laboratoire d'hydrobiologie de Toulouse avaient indiqué la 


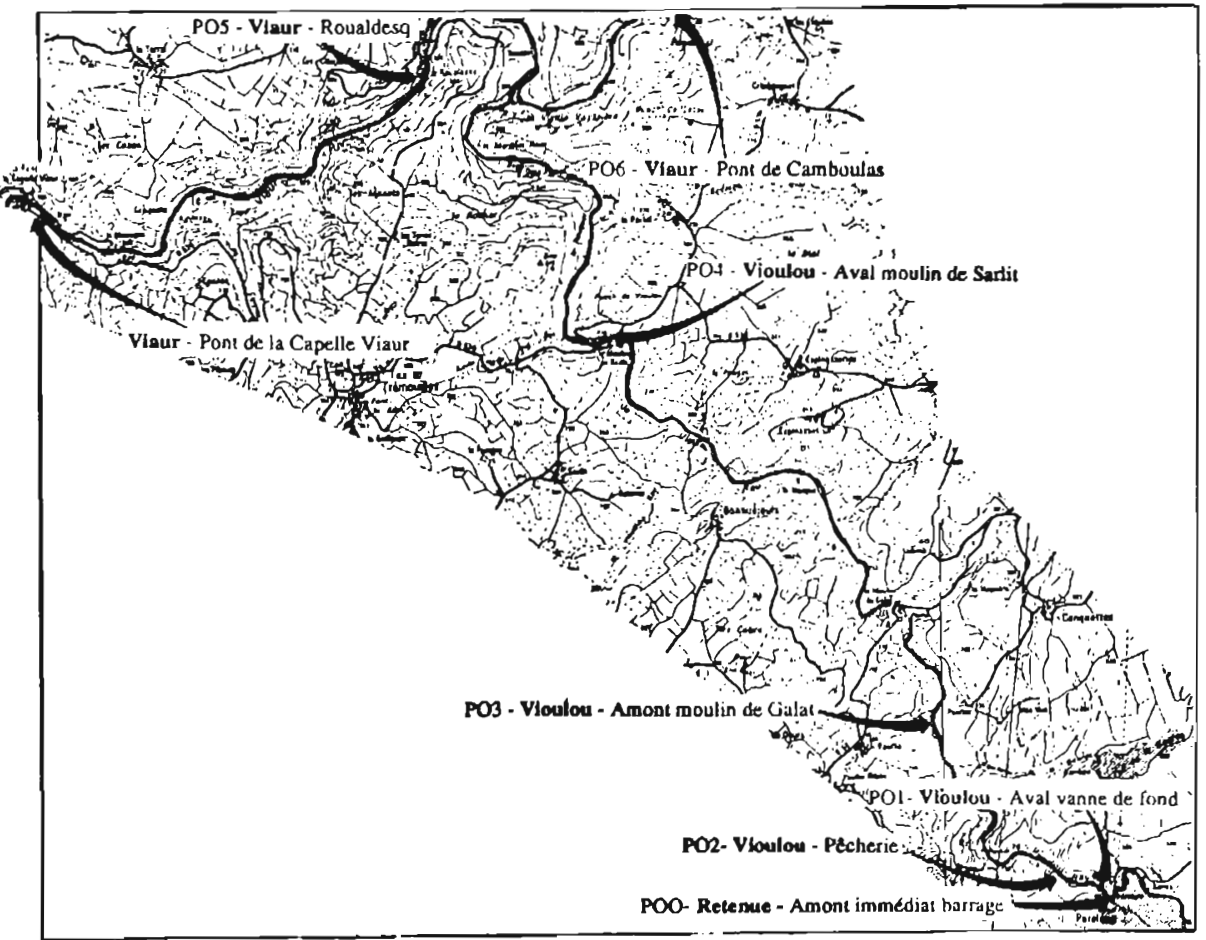

Fig. 4. - Plan de situation des points de mesure.

Fig. 4. - Reading sites.

présence de faibles épaisseurs de sédiments dans la retenue excepté à proximité immédiate des vannes de fond. Les sédiments sont assez sableux avec une consommation en oxygène limitée. Néanmoins, durant la dernière phase d'abaissement du plan d'eau, on ne peut éviter un lessivage partiel des rives avec un glissement des sédiments vers le barrage et le re-creusement du lit mineur du Vioulou. Les poissons, fortement concentrés avant d'être contraints à la dévalaison au travers de la vanne de fond, ont également contribué à remettre en suspension les sédiments.
Au début de la vidange, les teneurs mesurées à la pêcherie, soit $200 \mathrm{~m}$ en aval du barrage, sont restées assez faibles $(0,1 \mathrm{~g} / l)$ pour augmenter (fig. 5$)$ ensuite rapidement et se maintenir supérieures à $3 \mathrm{~g} / \mathrm{l}$ ce qui a conduit à diluer l'eau de la vidange par un apport de Bage jusqu'à la fin des opérations. Seuls quelques épisodes de courte durée en fin de passage du culot ont dépassé la valeur limite de $8 \mathrm{~g} / \mathrm{l}$.

Le flux total de sédiments au niveau de la pêcherie a été évalué à environ 7500 tonnes de matières sèches. 
A l'aval, plus on s'éloigne du barrage de Pareloup, plus la teneur en MES diminue ce qui indique qu'un phénomène de dépôt se produit auquel s'ajoute après le confluent avec le Viaur, une importante dilution entretenue par des lâchures constantes effectuées à Pont de Salars. Ceci s'explique par la nature des sédiments composés en grande partie de sables fins et grossiers qui se sont déposés dans les méandres du Vioulou. Seule la fraction la plus fine des MES a atteint le Viaur et a donné des élé- vations peu élevées de MES aux points de contrôle $A$ et $B$ (fig. 6).

\section{B) Oxygène dissous}

La teneur en oxygène dissous est restée supérieure à $6 \mathrm{mg} / \mathrm{l}$ au niveau de la pêcherie durant toute la vidange ce qui traduit la faible consommation en oxygène de sédiments. Au-delà, les teneurs ré-augmentaient rapidement montrant une bonne capacité de réoxygénation du Vioulou facilitée par le régime torrentiel du cours d'eau aux

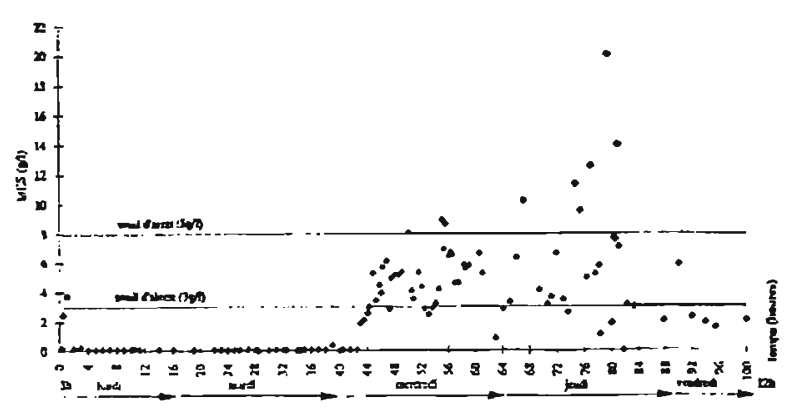

Fig. 5. - Evolution de la teneur en matieres en suspension $(g / l)$ à laval immédiat du barrage. Fig. 5. - Evolution of suspended solids (MES) concentrations $(g / /)$ immediately downstream of the dam.

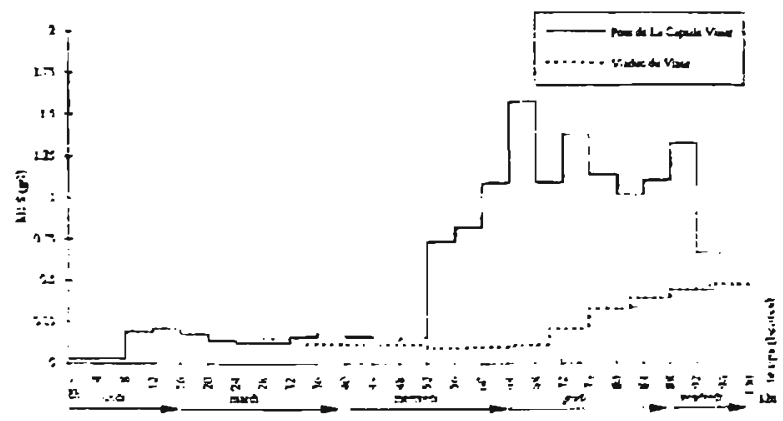

Fig. 6. - Evolution de la ten€ur en matieres en suspension ( $g / l$, aux points $A(4 \mathrm{~km})$ et $B(38 \mathrm{~km})$.

Fig. 6. -- Evolution of MES concentrations $(g / /)$ at points $A(4 \mathrm{~km})$ and $B(38 \mathrm{~km})$. 
débits qui ont été maintenus pendant la vidange. Aux points éloignés $A$ et $B$ sur le Viaur la baisse de teneur en oxygène dissous a été faible (de l'ordre de $1 \mathrm{mg} / \mathrm{l}$ ) et le pourcentage de saturation en oxygène du Viaur est resté supérieur à $90 \%$.

Dans la retenue par contre, la teneur en oxygène dissous a baissé rapidement à la fin du deuxième jour de vidange pour atteindre une anoxie presque complète le $3^{\mathrm{e}}$ jour. Pour éviter une mortalité massive des poissons qui n'avaient pas encore été entraînés par la vanne de fond jusqu'à la pêcherie, il a été décidé d'envoyer devant le barrage un lâcher d'eau claire et oxygénée pompée depuis Bage. Ceci a permis de terminer la vidange en limitant les échouages de poissons morts à quelques 200 mètres de part et d'autre du barrage (ramassage d'environ une tonne essentiellement de gardons et grosses brèmes).

\section{C) Azote ammoniacal}

Le relargage de l'ammoniaque $(\mathrm{N}$ $\mathrm{NH}_{4}$ ) est lié à un phénomène physique de désorption à partir des particules de sédiments. La cinétique de cette réaction est très rapide et on observe une montée progressive de la teneur en azote ammoniacal avant toute autre manifestation qualitative sensible des effets de la vidange.

Les teneurs en azote ammoniacal étaient déjà très élevées, de l'ordre de $1 \mathrm{mg} / \mathrm{l}$, avant l'ouverture de la vanne de fond et ont progressé jusqu'à plus de $3 \mathrm{mg} / \mathrm{l}$ à l'aval immédiat du barrage. Les valeurs élevées s'atté- nuaient progressivement vers l'aval en raison de la dilution du Viaur et en partie par l'oxydation de $\mathrm{NH}_{4}^{4}$ en $\mathrm{NO}_{2}^{-}$puis $\mathrm{NO}_{3}^{-}$(phénomène de nitrification) car les teneurs en oxygène dissous restaient importantes.

Même si la valeur impérative de la Directive Européenne sur la qualité des eaux à vocation salmonicole de $1 \mathrm{mg} / \mathrm{l}$ a été dépassée pendant toute la durée de la vidange, en raison du $\mathrm{pH}$ qui est resté neutre $(6,8$ à 7,2$)$ et de la bonne oxygénation des eaux, la fraction non ionisée de l'azote $\left(\mathrm{NH}_{3}\right)$ qui est toxique pour le poisson est restée très faible. La faune piscicole du Vioulou et du Viaur n'a semble-t-il pas été affectée et aucune mortalité de poisson n'a été observée sur les deux cours d'eau, ni dans une pisciculture privée du Pont de Sarlit (truites).

\section{D) Les métaux : Fer et Manganèse dissous}

Ces deux paramètres ont été suivis car des concentrations élevées (> $1 \mathrm{mg} / \mathrm{l}$ ) peuvent se révèler préjudiciables pour la production d'eau destinée à la consommation humaine (valeur guide Décret 91.257 du 7 mars 91).

Des concentrations assez fortes en fer, confirmées visuellement dans la retenue par la coloration rouge du substrat du lit sur lequel il y avait précipitation, (fig. 7) ont été mesurées dès le début du suivi $(4 \mathrm{mg} / \mathrm{l}$ dans la retenue amont, plus de $1 \mathrm{mg} / \mathrm{l}$ à l'aval). Ces valeurs élevées se sont maintenues durant toute la phase d'é- 


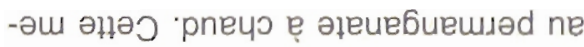

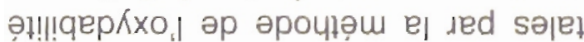
-ol səlqepkxo sadə!!eu səp eınsam

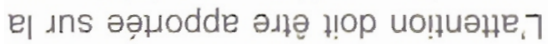

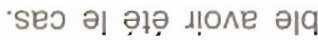

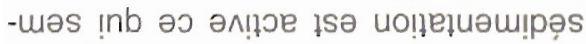

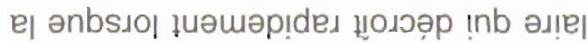

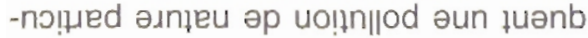
-!pu! |elol әגoudsoud ә $12000 \mathrm{R} 7$

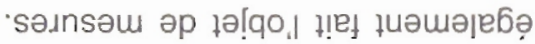
luo pletol aloudsoud 'sәtext!n 'sәlul

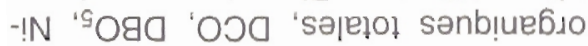

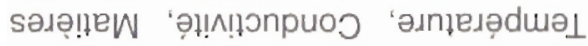

sanpuresed saınt (

-alqelod neap sabeldeo sal dnod

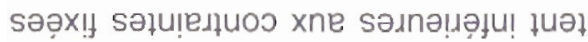

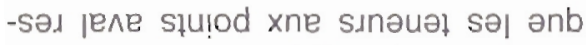

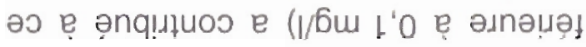

-u! uW uə ınəuə!) әsạuebuew ap nəd

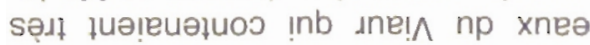
sə| led uo!̣n!!p eך 'әbuepı̊ el әp ınq

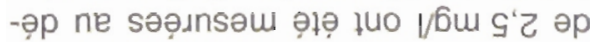

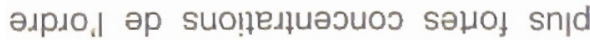

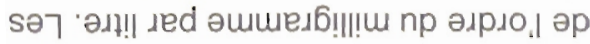

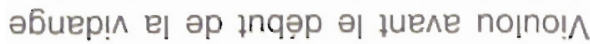

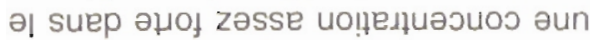

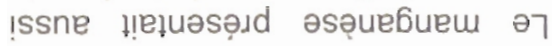

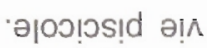
е| גns ґәщә,p snjd uou nә sed łuo,u

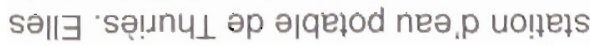

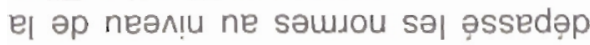
sed łuo u ła łuəss!̣eq sınauəן sa| ıne!^

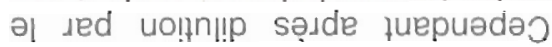

·łuenins

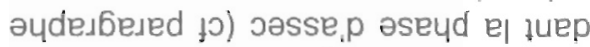

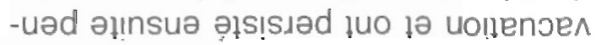

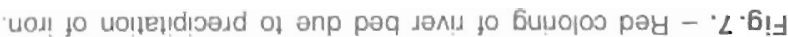
'

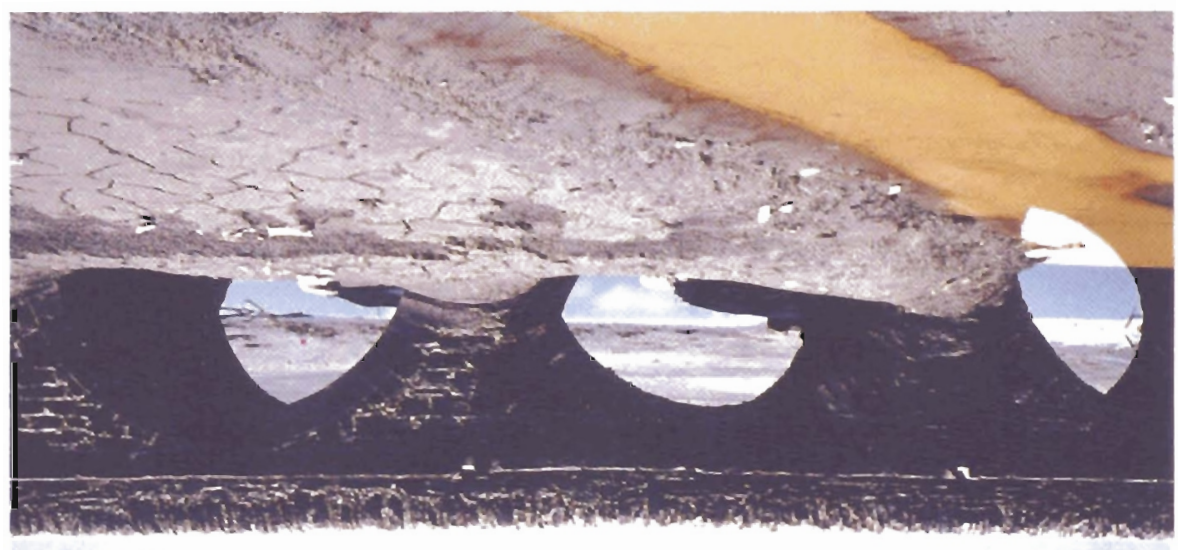


sure faisait l'objet d'une valeur limite de $10 \mathrm{mg} / \mathrm{l}$ dans l'arrêté préfectoral, qui est une valeur de référence pour le traitement des eaux brutes destinées à la fabrication d'eau potable.

La valeur de $10 \mathrm{mg} / \mathrm{l}$ a été dépassée avant l'ouverture des vannes de fond et des valeurs aberrantes allant jusqu'à $110 \mathrm{mg} / \mathrm{l}$ ont été constatées sur des échantillons prélevés au cours de la vidange, mais analysés en différé compte tenu des délais d'acheminement jusqu'au laboratoire et des temps d'analyse.

Lors de la vidange, les eaux sont fortement chargées en fines particules qui réagissent au permanganate, mais qui peuvent en grande partie être éliminées par une filtration à $2 \mu \mathrm{m}$ de l'eau (telle que le fait un filtre à sable à l'entrée d'une station de traitement d'eau potable). Des consignes de filtration soignée ayant été données aux différents traiteurs d'eau concernés, on n'a pas eu à constater une altération du fonctionnement des stations.

II s'avère donc que ce critère de qualité d'eau est peu adapté au suivi d'une vidange et doit au moins, s'il est réalisé, se faire sur une eau filtrée.

\section{2-3 La phase d'assec}

La phase d'assec a duré jusqu'au 17 septembre, date à laquelle les vannes de fond ont été refermées.

Durant cette phase, les travaux ont été réalisés sur le barrage.

- Un relevé des zones de sédimentation a été réalisé ainsi que des prélevements de sédiments.
Le bilan des dépots sédimentaires donne un rythme de $3429 \mathrm{t}$ par $\mathrm{km}^{2}$ et par an ce qui représente une épaisseur de $5 \mathrm{~mm}$ de dépots secs de caractéristiques physico-chimiques proches de celles décrites dans les milieux oligotrophes (Dagnac, 1994).

- La qualité de l'eau de Vioulou a continué à faire l'objet d'un suivi toutes les semaines en 4 points à l'aval du barrage pour les paramètres suivants: oxygène dissous, température, MES, $\mathrm{NH}_{4}$ et $\mathrm{Fe}$.

Dès début juillet, on assiste à un retour à la normale des paramètres de qualité d'eau avec des teneurs en MES inférieures à $0,1 \mathrm{~g} / \mathrm{l}$, une teneur d'oxygène dissous à saturation, environ $0,1 \mathrm{mg} / \mathrm{l}$ de $\mathrm{N}-\mathrm{NH}_{4}$, mais encore des teneurs en fer assez élevées jusqu'à la fin août de l'ordre de 0,8 à $1 \mathrm{mg} / \mathrm{l}$.

- Des pêches électriques ont eu lieu afin de déterminer la proportion de poissons réfugiés dans les principaux ruisseaux qui se jettent dans la retenue de Pareloup et qui serpentent dans la zone émergée.

C'est durant cette phase qu'ont eu lieu les travaux d'entretien et les principales animations touristiques du lac destinées à remplacer en partie les activités nautiques habituelles en période estivale.

\section{2-4 La phase de remise en eau}

Débutée en septembre 1993, elle s'est étalée sur toute la période pluvieuse jusqu'en mars 94. 
Le remplissage au départ s'est fait avec l'eau des retenues de Pont de Salars et de Bage dont la qualité n'est pas excellente en fin de période estivale (cyanophycées, manganèse...). Un suivi spécifique a été mis en place qui a montré que l'eau est plus conductrice et plus chargée en phosphore entrainant un enrichissement du lac de Pareloup en nutriments. Le ré-alevinage du lac est en cours sous la responsabilité de la DDAF avec la collaboration de la Fédération de pêche de l'Aveyron, de l'AAPP de Rodez et des scientifiques impliqués (introduction de salmonidés qui dominaient lors de la création de la retenue).

\section{LES MESURES D'ACCOMPAGNEMENT}

Les mesures d'accompagnement ont été définies avant la vidange et intégrées en partie dans l'arrêté préfectoral. Elles visent à minimiser les impacts de la vidange sur le tourisme, les autres usages de l'eau et l'environnement.

\section{3-1 Le tourisme et les loisirs}

Les mesures d'accompagnement dans ce domaine ont été très importantes lors de la vidange de Pareloup et toujours traitées par EDF en partenariat avec les collectivités locales et le SIVOM des lacs du LEVEZOU. II n'entre pas dans le cadre de cet article d'en détailler les actions.

\section{3-2 Les usages de l'eau}

Hormis le tourisme, les eaux du lac de Pareloup sont utilisées par le Syndicat des Eaux du SEGALA pour la production d'eau potable.

En anticipation de la vidange de Pareloup, EDF a étudié avec le Syndicat la possibilité d'une autre source d'approvisionnement. Une solution de remplacement a été mis en place par le Syndicat dès janvier 93 par pompage dans le lac de Bage.

En ce qui concerne les stations d'eau potable situées à l'aval, on a vu précédemment que la plus proche se situe à Pampelonne dans le Tarn (environ $40 \mathrm{~km}$ après Pareloup).Cette station a été très perturbée par la vidange de Thuriès en 1983. C'est la raison pour laquelle des mesures de surveillance particulieres ont été prises durant la vidange jusqu'à ce point (cf. 2.2).

\section{3-3 L'environnement aquatique}

Les principaux problèmes à résoudre concernaient le devenir des poissons présents dans le lac et la protection de la vie aquatique dans les cours d'eau à l'aval du barrage.

3-3-1 La récupération des poissons de la retenue

- L'ouvrage de pêcherie et l'organisation de la pêche, du tri et de l'évacuation des poissons selon leur destination ont été conçus, en collaboration étroite avec le Conseil supé- 
rieur de la Pêche, la Fédération de pêche de l'Aveyron et l'AAPP de Rodez, pour récupérer le maximum de poissons en quatre à cinq jours avec la mortalité la plus faible possible. Ceci a nécessité la mise en place de moyens très importants, tant matériels (10 bacs de stabulation de 1 à $2 \mathrm{~m}^{2}$, tables de tri, grue de chantier, bennes pour les poissons destinés à l'équarrissage, camions avec aérateurs pour les poissons transportés vivants...) qu'humains (équipes de pêcheurs se relayant $24 \mathrm{~h} / 24$ soit plus d'une centaine de personnes).

- Au début de la vidange du culot, le passage des poissons vers l'aval était relativement faible, puis s'est accéléré à partir du mardi soir avec environ 1 tonne/heure pour atteindre environ 3 tonnes/h le mercredi puis s'est affaibli rapidement le jeudi matin.

En début de vidange, tous les poissons ont été dénombrés, mesurés et pesés, ensuite les poubelles de transport ont été étalonnées en définissant des dominantes, la cadence de pêche ne permettant plus de peser chaque bac. Seuls les brochets, carpes, sandres, tanches, truites et chevesnes ont fait l'objet d'un dénombrement individuel durant toute la durée de la vidange.

Hormis, en début de vidange où les vitesses d'eau étaient importantes et où les poissons, (brèmes, gardons) étaient souvent écaillés, les poissons ont été capturés en très bon état physique et physiologique (Bau et al., 1994).

\section{A) Bilan au niveau de la pêcherie}

Au total, environ 67 tonnes de poissons ont été récupérées à la pêcherie. Les gardons dominent en masse, on trouve ensuite par ordre décroissant, les brèmes, les perches, les carpes, les brochets, les autres espèces étant beaucoup moins représentées.

En nombre d'individus, c'est également le gardon qui est l'espèce la plus représentée, suivie par les perches et les brèmes.

La destination des poissons fut la suivante:

- 43 tonnes (essentiellement des brèmes et des gardons) ont été transformées en farine de poisson.

- 3 tonnes ont été données aux Restos du cœur qui les ont commercialisées sur place;

- 21 tonnes ont été réparties entre les lacs aveyronnais et les Fédérations voisines pour le réalevinage.

A noter que 5 tonnes de gardons de plus auraient pu être conservés, mais il n'y avait plus de lieu de stockage possible.

\section{B) Autres captures}

Tous les poissons présents dans la retenue de Pareloup n'ont pas été capturés à la pêcherie.

- une partie des poissons s'est réfugiée lors de l'abaissement du culot dans la galerie d'Alrance, tunnel qui relie le lac de Pareloup au lac de Villefranche de Panat. Certains sont passés dans le lac de Villefranche mais la plupart a été piégée dans la galerie et a dû faire l'objet d'une ré- 
cupération spécifique du 28 juin au 2 juillet.

Environ 12,7 tonnes essentiellement des gardons et des brèmes ont été extraites de la galerie et détruites, auxquelles il faut ajouter environ 1,5 tonnes de brèmes ramassées dans le canal de fuite d'Alrance et quelques $250 \mathrm{~kg}$ de gardons sur le pourtour du lac de Villefranche (dont beaucoup d'alevins de 4 à $6 \mathrm{~cm}$ ).

- des pêches ont été effectuées durant la période d'abaissement du plan d'eau, pour environ 8 tonnes dont environ 2 tonnes ont été stockées dans la retenue des Faux, petite retenue collinaire de 2 ha créée spécifiquement pour la durée de la vidange dans une anse du lac alimentée par un ruisseau.

- quelques pêches scientifiques au filet ont encore été réalisées début juin (1 tonne stockée aux Faux) et pendant la fin de la vidange, de nombreux "pêcheurs" ont récupéré les poissons dans le culot. L'enquête réalisée in situ permet d'estimer le montant total de ces prises à 8 tonnes environ.

- des poissons se sont échoués ou ont été rejetés par les pêcheurs à proximité immédiate du barrage soit environ 2 tonnes de pertes essentiellement des brèmes et des gardons.

- enfin des poissons se sont réfugiés dans le Vioulou amont et les principaux ruisseaux qui alimentent la retenue de Pareloup. Les pêches électriques réalisées en ont dénombré environ 1 tonne. Le gardon est l'espece la plus abondante avec la perche mais un nombre important de truites, de chevesnes, de goujons et de vandoises, espèces peu représentées à la pêcherie a également été observé, ainsi que la présence de plusieurs milliers d'alevins.

En conclusion, on peut donc estimer à plus de 100 tonnes la population piscicole de la retenue de Pareloup avant vidange ce qui représente un peuplement particulièrement riche. Cette richesse et la multitude des interventions qui ont été pratiquées avant et pendant la vidange rendent cependant difficile l'établissement d'un bilan exact. On peut néanmoins dire, si on excepte le problème de la galerie d'Alrance (qui peut facilement être évité maintenant qu'il est connu), que les dispositifs de récupération étaient bien adaptés à une capture de poissons qui n'avait encore jamais été pratiquée à cette échelle et qu'ils ont permis la sauvegarde d'un cheptel piscicole important (de 21 tonnes rien que sur la pêcherie) permettant d'envisager des réalevinages conséquents avec des souches locales.

\section{3-3-2 l'écosystème aquatique aval}

Des mesures ont été prises à la fois pour préserver les rivières à l'aval du barrage et pour réaliser les observations nécessaires pour évaluer l'impact de la vidange sur le milieu aquatique.

Préalablement à la vidange, un relevé de l'état de la rivière (état des berges, envasement etc.), des indices biotiques et un inventaire piscicole ont été réalisés. 
Pour présenver la souche de truites fario, la Société de Pêche de Rodez a pratiqué des pêches électriques et a transféré le maximum de géniteurs dans le Vioulou en amont du barrage.

Globalement, il n'y a pas eu de grosse perturbation de l'écosystème aval lors de la vidange. II n'y a eu aucune mortalité piscicole même dans la pisciculture de truites du Moulin de Sarlit, située à $5 \mathrm{~km}$ du barrage. Le principal effet est une sédimentation en milieu lentique mais surtout formée de sables.

\section{- IBG}

Les mesures effectuées immédiatement après la vidange en juillet 93 accusaient une légère baisse par rapport à février 93 , touchant plus particulièrement les larves d'insectes vivant en milieu lentique et affectées surtout par l'augmentation de courant créée par le débit de vidange $\left(12 \mathrm{~m}^{3} / \mathrm{s}\right)$ dans un tronçon de rivière habituellement alimentée seulement par le débit réservé (Vergnes et al., 1993).

On notait également un apport temporaire de crustacés dérivants venant de la retenue.

Deux mois après la vidange, la recolonisation était déjà fortement amorcée sur les stations les plus éloignées du barrage et en février 94 , toutes les valeurs sont supérieures à celles notées en février 93 (15 à 17) avec des taxons identiques mais une densité et une biomasse plus élevées surtout en milieu lentique.

\section{- Population piscicole}

Les pêches électriques réalisées après la vidange par l'ENSA de Toulouse indiquent que quelques poissons de la retenue ont dévalé en aval du barrage sans doute après que l'on ait retiré les grilles de la pêcherie en fin de vidange du culot. On retrouve ainsi majoritairement des gardons (environ $100 \mathrm{~kg}$ sur l'ensemble du secteur d'étude) quelques brochets, perches, grémilles et vandoises. Les gardons sont souvent écaillés et amaigris, ce qui laisse à penser que la plupart ne survivra pas au delà de I'hiver 94.

En ce qui concerne les truites, les pêches ne sont pas le reflet de la situation initiale éventuellement influencée par la vidange puisque des prélèvements de truites ont été pratiqués entre temps. De plus un réalevinage en truites sauvages fario est prévu au printemps 94 .

\section{- Sédimentation - Frayères}

Les observations réalisées après vidange ont permis de constater qu'aucun dépôt de vase important n'a eu lieu à l'exception de la retenue du Moulin de Sarlit déjà très envasée avant la vidange. Ailleurs, le débit soutenu du Vioulou pendant la phase finale de vidange n'a permis que le dépôt des sables dans les méandres, zones qui pourraient peut-être devenir de nouvelles frayères.

$\mathrm{Ce}$ bon état constaté de la rivière associé à des valeurs d'IBG optimales a incité EDF à ne pas réaliser de chasses d'eau claire, chasses qui au- 
raient eu pour conséquence essentielle de lessiver de nouveau les invertébrés aquatiques qui ont parfaitement recolonisés le milieu et ce peu avant la période de frai de la truite.

\section{LE SUIVI DES CONSÉQUENCES À LONG TERME}

Après le remplissage à nouveau de la retenue. il s'avère très intéressant de poursuivre les études à la fois pour observer comment va se remettre en place l'écosystème lacustre et comment va évoluer le cours d'eau à l'aval du barrage. Les connaissances déjà acquises sur la retenue au cours des études hydrobiologiques qui ont précédé la vidange et l'intérêt que les chercheurs, notamment de Toulouse, portent à la poursuite des travaux sur ce site vont contribuer à acquérir des données précieuses sur les conséquences à long terme des vidanges, données très fragmentaires jusqu'à présent.

\section{1 à l'aval du barrage}

La mesure des indices biotiques va se poursuivre pendant 3 ans après la vidange à raison d'une mesure par saison sur les 4 stations choisies à l'aval du barrage. Indirectement, ces mesures seront l'occasion de suivre l'évolution des dépôts de sable.

En ce qui concerne les poissons. une pêche électrique aura lieu en 1994 qui permettra essentiellement de suivre l'élimination des espèces apportées par la vidange, mais le suivi ne sera pas poursuivi au-delá puisque ce secteur subit une pression halieutique variable et des réalevinages. La surveillance scientifique d'un secteur aval ne présente un intérêt que si la population initiale reste en place et si la gestion du cours d'eau est parfaitement contrôlée.

\section{2 dans la retenue}

Le réservoir de Pareloup est le site idéal pour étudier l'effet de discontinuité introduit par une vidange sur un écosystème lacustre à l'échelle de plusieurs années. En effet, dans le cadre d'une convention avec le Ministère de l'Environnement, des campagnes de mesures lourdes ont été menées de 1986 à 1989 puis exploitées par l'UPS et le groupe Gestion de l'Eau et Environnement de la Direction des Etudes et Recherches d'EDF (GEE) pour mettre au point un modèle numérique de l'écosysteme de Pareloup (Salençon et Thébault, 1994). On a donc une assez bonne connaissance du fonctionnement de l'écosysteme de ce lac pendant près de 30 ans (entre la vidange de 1962 et celle de 1993) notamment en ce qui concerne le plancton et le cycle du phosphore.

Un programme d'études portant sur les premieres années suivant le remplissage a donc été défini entre l'UPS et le GEE comprenant : 
- des prélèvements d'eau et des analyses in situ, assurés avec une fréquence régulière,

- des programmes de mesures spécifiques in situ et en laboratoire par l'UPS pour étudier certains poins particuliers comme l'évolution de la densité du plancton et des poissons, le comportement des sédiments et des végétaux submergés après le remplissage...

A partir de ces mesures, le modèle numérique existant sera utilisé afin de vérifier ses capacités à reproduire les évolutions que l'on constatera dans les années 1994 et 1995, fortement déficitaires en biomasse pisciaire comparativement à l'avant-vidange. Ensuite, soit le modèle sera validé si les écarts calculs-mesures sont faibles et on pourra envisager de l'utiliser à l'occasion d'études sur d'autres sites, sinon une phase de perfectionnement sera nécessaire.

\section{BILAN ET RETOUR D'EXPÉRIENCE POUR D'AUTRES VIDANGES}

Le bilan de la vidange de Pareloup peut, sans fausse modestie, être avant tout celui du succès d'une opération délicate et de grande envergure qui s'est déroulée conformément à ce que l'on avait prévu en informant en permanence le public, les médias et les nombreux collaborateurs d'EDF (élus, administrations, scientifiques) de l'évolution des opérations.
Cette réussite n'est pas le fruit de la chance, mais celui d'une préparation très soignée qui a débutée plus de 3 ans avant la date de la vidange en privilégiant la concertation et aussi celui de la connaissance exceptionnelle de l'écosystème de ce lac que l'on possédait grâce à la présence permanente de chercheurs sur le site depuis plus de 10 ans.

En contrepartie, la renommée de ce lac tant du point de vue scientifique que touristique et sa taille $\left(5^{\mathrm{e}}\right.$ retenue hydroélectrique de France) ont amené à réaliser des études ou des opérations (information, tourisme notamment) qui ne sont pas nécessairement adaptées ou réalisables Iorsqu'on vidange une retenue de moindre envergure. Néanmoins les acquis sont indéniables et peuvent être classés en trois types.

- ceux qui permettent d'expliquer des phénomènes ou des évolutions particulières d'un compartiment de l'écosystème lors des vidanges. II s'agit par exemple des observations qui ont été faites sur le comportement des sédiments (Labroue et al., 1994) ou sur le stress physiologique des poissons lors de pêches (Bau et al., 1994).

- ceux qui démontrent ou non la pertinence de l'utilisation de tel ou tel procédé ou de telle méthode. Ce sont en quelque sorte "les exemples à reprendre". Dans le cas de la vidange de Pareloup, ce sont surtout les acquis au niveau de la conception et de l'efficacité de la pêcherie qui sont importants. Mais d'autres points sont également très intéressants comme : 
- les possibilités d'utiliser l'écho-intégration pour évaluer le stock piscicole présent dans la retenue avant la vidange,

- la possibilité de créer une petite retenue de stockage du poisson dans une anse de la retenue,

- l'apport indéniable du suivi en temps réel des principaux paramètres de qualité d'eau pour piloter les manœuvres d'abaissement du plan d'eau, à condition de bien choisir le pas de temps de mesure et le point de mesure,

- l'intérêt de disposer de moyens de dilution aussi bien à l'amont qu'à l'aval du barrage,

- limportance du choix de paramètres de contrôle adaptés au cas des vidanges (cas de la mesure d'oxydabilité),

- le relevé des zones et l'analyse de la nature des sédiments déposés dans la retenue pendant la phase d'assec qui permet plus aisément de faire un bilan sédimentaire et d'avoir des éléments de référence précis permettant par la suite de suivre l'évolution de la sédimentation par des méthodes indirectes (bathymétries, échosondages...)..

- et bien que l'on ne les ait pas abordées ici la stratégie de communication et la constitution de la cellule de pilotage,

- enfin ceux qui concernent les mesures qui n'ont pas été prises ou faites et pour lequel le retour d'expérience montre qu'elles auraient été utiles ou qu'elles le seront pour d'au- tres vidanges soit à Pareloup, soit ailleurs.

Elles concernent essentiellement les deux points qui sont parmi les plus déterminants dans une vidange à savoir les sédiments entraînés pendant la vidange et la récupération des poissons.

Pour les sédiments, il apparaît certain que les impacts sur la vie aquatique et même les usages de l'eau comme la fabrication d'eau potable n'ont pas la même ampleur si l'on a affaire à des sédiments très sablonneux, peu consommateurs d'oxygène et qui décantent vite ou s'il s'agit de vases très chargées en matières organiques avides d'oxygène.

II importe à l'avenir de s'attacher à mettre en œuvre des méthodes de mesure qui permettent de différencier les différentes fractions qui composent les sédiments qui vont transiter vers l'aval et d'évaluer leur temps de redéposition. Plusieurs moyens pourraient être utilisés séparément ou simultanément comme :

- prendre un point de mesure pour piloter la vidange plus à l'aval du barrage (à environ $1,5 \mathrm{~km}$ dans le cas de Pareloup) afin d'avoir une homogénéisation des sédiments dans la veine d'eau rejetée par la vanne de fond et déjà une décantation de la plupart des sables grossiers.

- effectuer des mesures de MES sur une eau filtrée à $50 \mu \mathrm{m}$ pour séparer les sables de la fraction vaseuse.

- utiliser un granulomètre à laser, appareil qui peut, dans certains cas, 
être déplacé sur le terrain dans un camion-laboratoire.

Enfin, pour mieux connaître les volumes, l'emplacement et le comportement des sédiments qui vont se déposer à l'aval, il faut réaliser un repérage précis de l'état de sédimentation du tronçon aval qui sera concerné par la vidange, notamment au niveau des zones de sédimentation potentielles et des frayères.

Pour les poissons, il s'est avéré à Pareloup qu'il est nécessaire lorsque les poissons commencent à être concentrés dans un volume d'eau restreint, de disposer de mesures de qualité d'eau concernant le culot afin de mieux comprendre leur comportement et d'anticiper les risques de mortalité. Des moyens adéquats doivent être prévus pour récupérer le poisson devant le barrage lorsque la concentration devient trop forte et/ou que les poissons ne dévalent pas par les orifices de fond.

A Pareloup, une grande partie de cette récupération a été réalisée par des "pêcheurs" occasionnels profitant de l'aubaine dans des conditions de sécurité très relatives. Pour éviter cela, il serait souhaitable de confier cette pêche finale à un professionnel ce qui s'est déjà pratiqué pour les vidanges d'autres retenues.

Les poissons présents avant la vidange ont été ré-implantés dans d'autres lacs de la région, avec des taux de succès très élevés (mortalités insignifiantes). Certains comme ceux stockés dans la retenue de Faux vont retrouver le lac. Mais on souhaite re- donner à Pareloup une population à dominante salmonicole et les re-alevinages vont être orientés dans ce sens. Le plan de gestion piscicole doit être rigoureux et bien connu des scientifiques si on veut comprendre d'ici à quelques années l'évolution du cheptel piscicole. Bien que cela ne soit pas du ressort d'EDF, il semble utile que des plans de gestion piscicole clairs soient établis pour les retenues après vidange.

En conclusion, à Pareloup, la vidange de la retenue en 1993 a été menée avec une large concertation et un recours important aux scientifiques et techniciens connaissant bien l'écosystème de Pareloup et notamment sa richesse piscicole ce qui a permis à la fois de réussir une opération qui est toujours délicate sans porter atteinte à l'environnement aquatique et d'enrichir l'expérience acquise dans le domaine des vidanges. Les études qui se poursuivent sur les effets à long terme apporteront encore des éléments précieux sur les capacités de régénération du milieu après une étape aussi radicale dans la vie du lac.

\section{REMERCIEMENTS}

La vidange de la retenue de Pareloup n'aurait pas pu être menée à bien sans le dévouement de tous ceux, scientifiques, techniciens, étudiants et bénévoles qui y ont apporté leur concours avec compétence et enthousiasme.

Qu'ils en soient ici tous sincèrement remerciés. 


\section{RÉFÉRENCES}

Andrieu J.P., 1993. Résultats du suivi des principaux paramêtres physico-chimiques. Vidange de la retenue de Pareloup sur le Vioulou. Juin 1993. Rapport EDF/DTG-RE/VID0054A.

Bau F., Parent J.P., Vellas F., 1994. Influence de la vidange d'une retenue sur certaines caractéristiques physiologiques chez divers Téléostéens. Hydroécol. Appl 6 (1/2) : pp. 293-314.

Cardinal H., 1988. Bilan des recherches et recommendations en matière de vidanges de retenues. Procédure LIVRE. Rapport EDF/DER-HE-31/88-20.

Dagnac J., 1994. Bilan des dépots sédimentaires dans le réservoir de Pareloup (Aveyron, France). Hydroécol. Appl. 6 (1/2) : pp. 59-85.

Energie Midi-Pyrénées, 1994. Présentation de l'aménagement hydroélectrique du Pouget el du barrage de Pareloup (Aveyron). EDF Production Transport. Hydroécol. Appl. 6 (1/2) : pp. 1-7.

Labroue L., Capblancq J., Salençon M.J., Tourenq J.N., Mur C., 1994. Evolution saisonnière des éléments minéraux nutritifs ( $P, N, S i)$ et de l'oxygène dis- sous dans le lac de Pareloup. Hydroécol. Appl. 6 (1/2) : pp. 87-114.

Richeux Ch., Nogués J.F., Tourenq J.N., Aragon B., 1994. Inventaire piscicole de la retenue hydroélectrique de $\mathrm{Pa}$ reloup (Aveyron) lors de la vidange de Juin 1993. Essai d'un nouveau système d'acquisition et de traitement des signaux d'un échosondeur. Hydroécol. Appl. 6 (1/2): pp. 197-226.

Salençon M.J. et Thébault J.M., 1994. Modélisation de l'écosysteme du Lac de Pareloup avec les modèles ASTER et MELODIA. Hydroécol. Appl. 6 (1/2): pp. 369-426.

Simonet F.. Ferroni J.M., Tourenq J.N., 1993. Suivi de l'impact de la vidange de la retenue de Pareloup sur la qualité des eaux du Viaur (14-18 Juin 1993). Agence de l'eau Adour Garonne et Laboratoire d'Hydrobiologie (URA 695) de I'UPS de Toulouse. Oct. 1993. 18 pages.

Vergnes L., Tourenq J.N., 1993. Etude de l'impact de la vidange du barrage de Pareloup sur la faune benthique du Vioulou et du Viaur (Aveyron). Mémoire de D.E.S.U. Laboratoire d'Hydrobiologie URA 695. Université Paul Sabatier de Toulouse. 